\title{
Administração oral de piperina em frangos de corte
}

\author{
Piperine oral administration on broiler' chikens
}

\author{
Verônica da Silva CardosoI Cristina Amorim Ribeiro de Lima $^{\text {II }}$ Marco Edílson Freire de Lima ${ }^{\text {III }}$ \\ Luis Eduardo Gomes Dorneles ${ }^{\mathrm{IV}}$ Walter Leira Teixeira Filhov ${ }^{\mathrm{V}}$ Raquel Silva Lisboa $^{\mathrm{I}}$ \\ Daniel da Silva Guedes Junior ${ }^{I}$ Glória Maria Direitov \\ Maria das Graças Miranda Danelli ${ }^{\mathrm{*}}$
}

RESUMO

O efeito tóxico da piperina, principal amida presente em diversas espécies de pimenta, foi avaliada em frangos de corte por meio da administração oral $(0,00,1,12$, 2,25 e 4,5 mg kg-1 peso vivo) por 14 dias consecutivos. Foram empregados 60 pintos machos (Cobb Avian 48) com sete dias de idade, distribuídos aleatoriamente em quatro grupos experimentais $(n=15)$. Foram avaliados parâmetros como: ganho de peso, peso relativo do fígado e alterações hematológicas e anatomopatológicas. A administração oral de piperina não interferiu no ganho de peso ou no peso relativo do fígado, além de não promover alteração no tamanho e na coloração dos órgãos ou no aparecimento de lesões de parênquima e nas mucosas do órgão. Todavia, alterações histopatológicas dose-dependentes foram observadas. A piperina não foi capaz de alterar significativamente os parâmetros hematológicos analisados, com exceção do leucograma, em que as doses de $1,12 \mathrm{mg}$ e $2,25 \mathrm{mg} \mathrm{kg}^{-1}$ promoveram aumento do número de heterófilos e do número total de leucócitos, respectivamente. Os resultados sugerem que a dose oral de 1,12mg de piperina por quilo não é tóxica para frangos de corte, sendo semelhante aos resultados obtidos para ratos e camundongos. Além disso, constatou-se a capacidade da piperina em aumentar o número total de leucócitos circulantes a partir da dose de 2,25 $\mathrm{mg} \mathrm{kg}^{-1}$ nessa espécie animal.

Palavras-chave: frangos de corte, piperina, toxicidade.

\begin{abstract}
The toxic effect of piperine, the main amide compound of different pepper species, was evaluated on broiler chickens by oral administration at 0.00,1.12, 2.25 and $4.50 \mathrm{mg}$ $\mathrm{kg}^{-1}$ concentrations for 14 days. Sixty seven days old male chicks (Cobb Avian 48) randomly allocated to four experimental groups $(n=15)$ were used in this work. Parameters such as: body weight gain, liver relative weight, hematological and anatomopathological alterations were analyzed. The oral route administration did not interfere on weight gain or liver relative weight, as well as, any modification on size and organs' color and/or parenchyma/mucous membranes injuries were observed; however, liver histopathological changes were noticed in a dose-dependent manner. In addition, piperine did not alter hematological parameters, except for leukocytes counting, which at 1.12 and from $2.25 \mathrm{mg} \mathrm{kg}^{-1}$ caused an increase of heterophils and in the total number of leukocytes, respectively. The results suggest that $1.12 \mathrm{mg} \mathrm{kg}^{-1}$ of piperine orally administrated is not toxic for broiler chickens, as previously observed for rats and mice. Moreover, $2.25 \mathrm{mg} \mathrm{kg}^{-1}$ of piperine seems to increase the total number of leukocytes for this animal specie.
\end{abstract}

Key words: broiler chicken, piperine, toxicity.

\section{INTRODUÇÃO}

Piperina (1-piperoil piperidine) é o componente ativo presente em diversas espécies de

\footnotetext{
IPrograma de Pós-graduação em Ciências Veterinárias, Universidade Federal Rural do Rio de Janeiro (UFRRJ), Seropédica, RJ, Brasil.

IIInstituto de Zootecnia, UFRRJ, Seropédica, RJ, Brasil.

III Instituto de Ciências Exatas (ICE), UFRRJ, Seropédica, RJ, Brasil.

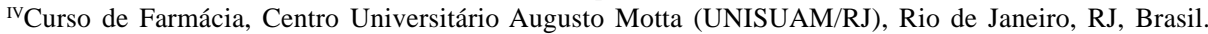

v Departamento de Microbiologia e Imunologia Veterinária, Instituto de Veterinária, UFRRJ, BR 465, km 7, 23890-000, Seropédica, RJ, Brasil. E-mail danelli@ufrrj.br.*Autor para correspondência.
} 
pimenta (Piper sp.), usadas mundialmente como temperos e como ingrediente em sistemas de medicina alternativa (PARMAR et al., 1997). Essa amida é um composto lipofílico absorvida no intestino e rapidamente biotransformada no fígado, pela demetilação do seu grupamento metilenodióxido (BHAT \& CHANDRASEKHARA, 1986). Sua biotransformação é realizada, principalmente, pelo sistema microssomal do citocromo P-450 dos hepatócitos, sendo totalmente biotransformada e rapidamente excretada, sem deixar resíduos detectáveis nos tecidos após 24 horas da sua ingestão (BHAT \& CHANDRASEKHARA, 1986).

Diversas atividades biológicas e farmacológicas da piperina já foram demonstradas em animais de laboratório e em humanos. A suplementação de piperina promove atividade antiparasitária (GHOSHAL et al., 1996), antipirética, analgésica (PARMAR et al., 1997), anti-inflamatória (GUPTA et al., 2000), contraceptiva, antiespermatogênica (MALINI et al., 1999), antioxidante (MITTAL \& GUPTA, 2000), inseticida (PARMAR et al., 1997; ESTRELA et al., 2003), imunomoduladora (SUNILA \& KUTTAN, 2004), anticarcinogênica (PRADEEP \& KUTTAN, 2002; SELVENDIRAN et al., 2004; SUNILA \& KUTTAN, 2004) e quimioprotetora (PATHAK \& KHANDELWA, 2007). Além disso, a piperina aumenta a biodisponibilidade de algumas substâncias; ela inibe a biotransformação hepática e intestinal de certas drogas como no caso do curcumin (SHOBA et al., 1998), do propanolol e da theofilina (BANO et al., 1991) e de outras drogas e outros suplementos nutritivos. A piperina atua inibindo diferentes isoformas do citocromo P-450 e outras enzimas envolvidas no metabolismo de xenobiótico, como UDP-glicorunil transferase e arilhidrocarbono hidroxilase hepática (ATAL, et al., 1985; BADMAEV et al., 1999). ATAL et al. (1985) sugerem ainda que a piperina prejudica a biotransformação de pró-carcinógenos e, portanto, a geração de seus intermediários reativos. Com base nessas observações, REEN et al. (1997) demonstraram a redução da citotoxicidade e a genotoxicidade da aflatoxina $\mathrm{B}_{1}$ in vitro, proporcionando um potente efeito quimiopreventivo contra pró-carcinógenos ativados pelo citocromo P-450 em hepatócitos de rato in vitro.

Diversos aspectos contribuem para o interesse pelo potencial da piperina como aditivo natural no setor avícola: (i) é um produto natural isolado em grande quantidade; (ii) tem baixo custo de produção; (iii) não produz acúmulo de resíduos detectáveis no organismo animal; (iv) possui propriedade citoprotetora, demonstrando claramente sua capacidade antioxidativa, antiapoptótica e de restauração de células com respostas mitogênicas proliferativas ou alterações fenotípicas, sugerindo seu uso terapêutico em condições de comprometimento do sistema imunológico de mamíferos.

Dessa forma, o excelente espectro de importância terapêutica da piperina em mamíferos credencia o estudo dessa amida no modelo aves. O presente estudo teve como objetivo verificar o efeito tóxico da piperina sobre diversos tecidos e diferentes células sangüíneas em frangos de corte.

\section{MATERIAL E MÉTODOS}

Piperina: purificada a partir dos frutos secos de Piper nigrum, empregando etanol como solvente, conforme descrito por IKAN (1991). A amida natural foi obtida com rendimento de 5-7\% e com elevado grau de pureza ( $\sim 98 \%$, determinado por GC-MS). O produto isolado mostrou ponto de fusão $\left(128-129^{\circ} \mathrm{C}\right)$ e dados espectrométricos $\left({ }^{1} \mathrm{H}\right.$ and ${ }^{13} \mathrm{C}$ NMR, IR and MS) idênticos aos reportados na literatura (ARAUJOJUNIOR et al., 1997; SIDDIQUI et al., 1997).

Aves e tratamento: 60 pintos machos, com sete dias de idade, da linhagem de corte Cobb Avian 48 foram separados em quatro boxes $(n=15)$ e mantidos sob condições ambientais do tipo padrão, com luz natural, recebendo ração balanceada e água ad libitum, de modo a atender as exigências nutricionais de ROSTAGNO et al. (2005). Os grupos experimentais foram divididos da seguinte forma: grupo1, animais inoculados apenas com o veículo empregado na diluição da piperina; grupo 2, 3 e 4, animais inoculados com piperina nas concentrações de 1,12, 2,25 e 4,50 $\mathrm{mg} \mathrm{kg}^{-1}$ de peso vivo, respectivamente. A administração dos tratamentos foi por via oral (gavagem), por 14 dias consecutivos. As doses empregadas foram escolhidas tendo como base o trabalho realizado por PIYACHATURAWAT et al. (1983), as quais representam, respectivamente, 1/8, 1/4 e 1/2 das doses geralmente usadas no tratamento de humanos (9-13mg $\left.\mathrm{kg}^{-1}\right)$.

Desenho experimental: ao final de 14 dias de experimento, os frangos com 21 dias de idade foram pesados e abatidos segundo normas estabelecidas pela instrução normativa $\mathrm{n}^{0}$ 3, de 17 de janeiro de 2000, do Ministério da Agricultura Pecuária e Abastecimento (http:/extranet.agricultura.gov.br/sislegis-consulta/ consultarlegislacao.do). O ganho de peso foi obtido pelo cálculo da diferença entre o peso das aves no início e ao final do experimento. Fígado, proventrículo, moela, duodeno, jejuno, íleo e ceco de todos os grupos foram avaliados macroscopicamente quanto à coloração, à forma, ao tamanho, à consistência, ao odor 
e à presença ou não de lesões ao exame visual e, posteriormente, foram coletados e fixados em formalina tamponada a 10\% (pH 7,8). Duas secções dos tecidos foram processadas de acordo com técnicas histopatológicas usuais, sendo os tecidos corados com hematoxilina-eosina, de acordo LUNA (1968). Os cortes histológicos foram observados ao microscópio óptico e classificados quanto à estimativa do grau da severidade da lesão (ausente, discreta, moderada e grave). Amostras de sangue foram coletadas com EDTA, por meio de venopunção basílica (veia alar), no início e ao final do experimento para a realização das análises hematológicas: concentração de proteínas plasmáticas totais (PPT), determinada por meio do método de refratometria (COLES, 1984); concentração de hemoglobina, dosada por meio do método de oxihemoglobina e centrifugação da amostra a 1.000xg por 10 minutos para a determinação da densidade óptica no fotocolorímetro (Klett Summersom ${ }^{\circledR}$ ) e correção do valor para a unidade de hemoglobina (CAMPBELL \& DEIN, 1984); determinação do fibrinogênio e hematócrito efetuadas por meio do método do microhematócrito segundo metodologia descrita por JAIN (1993); por meio de fórmulas padronizadas, foram calculados os seguintes índices: volume globular médio (VGM) e concentração de hemoglobina globular média (CHGM) (WINTROBE, 1933). As contagens de hemácias e leucócitos totais foram realizadas em hemocitômetro, utilizando-se a solução de Natt e Herrick (NATT \& HERRICK, 1951). A contagem diferencial de leucócitos foi realizada por meio de esfregaços sangüíneos corados pelo Panótico Rápido ${ }^{\circledR}$.

Análise Estatística: foi utilizado um delineamento inteiramente casualizado, sendo os resultados expressos pela média \pm desvio padrão e avaliados por meio da análise de variância paramétrica (ANOVA), empregando o programa GraphPad Prism 4.0 (2003). Quando diferenças estatísticas foram observadas, foi aplicado o teste de Newman-Keuls em nível de 5\%.

\section{RESULTADOS E DISCUSSÃO}

Estudos iniciais sobre a toxicidade da piperina, por diferentes vias (intravenosa, intraperitoneal, subcutânea, intramuscular e intragástrica), em camundongos, ratos e hamsters foram realizados, em 1983, por PIYACHATURAWAT et al. Segundo os autores, a administração de piperina por via intra-gástrica é bem tolerada em camundongos machos adultos com $\mathrm{DL}_{50 \%}$ elevada (330 $\mathrm{mg} \mathrm{kg}^{-1}$ ). DOGRA et al. (2004) investigaram o efeito da piperina em camundongos swiss machos por cinco dias consecutivos, estabelecendo a dose oral de 1,12mg $\mathrm{kg}^{-1}$ como segura para a espécie; dose equivalente a aproximadamente $1 / 8$ da dose geralmente usada no tratamento de humanos. Resultados semelhantes foram obtidos no nosso laboratório, com ratos machos adultos, em que a administração oral de piperina, em doses superiores a $1,12 \mathrm{mg} \mathrm{kg}^{-1}$ (2,25 e 4,50mg kg-1), promoveu alterações anatomopatológicas ou hematológicas significativas, compatível com intoxicação dose-dependente (Dra. Maria das Graças Danelli, comunicação pessoal).

As doses de piperina (1,12, 2,25 e 4,5mg kg-1 peso vivo) instituídas nesta avaliação não promoveram morte ou alterações clínicas no estado geral dos animais. Os animais não apresentaram alterações no ganho de peso (Tabela 1) ou sinais clínicos de intoxicação em nenhum dos grupos experimentais, sendo a piperina, aparentemente, bem tolerada em frangos de corte. As avaliações anatomopatológicas realizadas no momento do abate não revelaram alterações no tamanho, na forma, na consistência e na coloração dos órgãos ou nas lesões de parênquima e nas mucosas. A histopatologia revelou ausência de lesões no proventrículo, duodeno, jejuno, íleo e ceco, em todos os grupos experimentais. No tecido hepático, entretanto, foram detectadas lesões na zona 2 do ácino de Rapapport, que variaram de discreta à grave e estão mostradas na tabela 2: infiltrado multifocal de células mononucleares, predominantemente linfócitos, estendendo-se ao parênquima hepático em todos os grupos, sendo de grau discreto no grupo controle e grupo 2 (1,12 $\left.\mathrm{mg} \mathrm{kg}^{-1}\right)$, moderado no grupo 3 (2,25mg $\mathrm{kg}^{-1}$ ) e grave no grupo $4\left(4,5 \mathrm{mg} \mathrm{kg}^{-1}\right)$. Este último grupo exibiu ainda desarranjo trabecular e áreas de necrose inicial do epitélio dos ductos biliares. Esteatose também foi observada com o aumento da dose de piperina, sendo discreta e moderada nas doses de 2,25 e 4,50, respectivamente. Outras alterações importantes no tecido hepático foram observadas: discreta e moderada degeneração vacuolar do tecido hepático, congestão e proliferação das células epiteliais dos ductos biliares nas amostras dos grupos que receberam 2,25 e 4,50mg $\mathrm{kg}^{-1}$, respectivamente, caracterizando um quadro de intoxicação dose-dependente. Resultados preliminares obtidos por CARDOSO (2007) indicaram ainda que a piperina não foi capaz de interferir na conversão alimentar ou no consumo médio de ração de frangos de corte.

Os efeitos da administração oral de piperina sobre os parâmetros hematológicos em frangos de corte são apresentados na tabela 1 . O hemograma não revelou diferença significativa $(\mathrm{P}<0,05)$ entre os grupos experimentais, expressando valores hematológicos 
Tabela 1 - Efeito da administração de piperina em frangos de corte com 21 dias.

\begin{tabular}{|c|c|c|c|c|}
\hline \multicolumn{5}{|c|}{ Piperina (mg kg ${ }^{-1}$ ) } \\
\hline Parâmetros & 0,00 & 1,12 & 2,25 & 4,50 \\
\hline Viabilidade (\%) & 100 & 100 & 100 & 100 \\
\hline Ganho de peso (g) & $824^{\mathrm{a}} \pm 32,82$ & $786,9^{a} \pm 43,62$ & $842,5^{\mathrm{a}} \pm 43,75$ & $789,0^{a} \pm 34,12$ \\
\hline PRF (mg 100g $\left.{ }^{-1}\right)$ & $2,35^{\mathrm{a}} \pm 0,19$ & $2,36^{\mathrm{a}} \pm 0,19$ & $2,31^{\mathrm{a}} \pm 0,23$ & $2,27^{\mathrm{a}} \pm 0,15$ \\
\hline Hemácias $\left(\mathrm{x} 10^{6} \mathrm{~mm}^{-3}\right)$ & $2,219^{a} \pm 0,20$ & $2,193^{\mathrm{a}} \pm 0,20$ & $2,229^{a} \pm 0,10$ & $2,318^{a} \pm 0,25$ \\
\hline Hematócrito (\%) & $31,53^{\mathrm{a}} \pm 2,94$ & $32,71^{\mathrm{a}} \pm 2,40$ & $32,33^{\mathrm{a}} \pm 1,49$ & $32,33^{\mathrm{a}} \pm 2,25$ \\
\hline Hemoglobina $\left(\mathrm{g} \mathrm{dL}^{-1}\right)$ & $7,96^{\mathrm{a}} \pm 0,87$ & $8,24^{\mathrm{a}} \pm 0,78$ & $8,31^{\mathrm{a}} \pm 0,76$ & $7,87^{\mathrm{a}} \pm 0,73$ \\
\hline $\mathrm{MCV}(\mathrm{fL})$ & $142,5^{\mathrm{a}} \pm 11,69$ & $149,8^{\mathrm{a}} \pm 10,21$ & $147,0^{\mathrm{a}} \pm 7,26$ & $139,5^{\mathrm{a}} \pm 16,03$ \\
\hline $\operatorname{MCHC}\left(\mathrm{g} \mathrm{dL}^{-1}\right)$ & $25,08^{a} \pm 1,22$ & $25,20^{\mathrm{a}} \pm 1,94$ & $25,70^{a} \pm 2,15$ & $24,43^{\mathrm{a}} \pm 2,60$ \\
\hline $\mathrm{PPT}\left(\mathrm{g} \mathrm{dL}^{-1}\right)$ & $2,620^{a} \pm 0,31$ & $2,557^{\mathrm{a}} \pm 0,17$ & $2,793^{a} \pm 0,26$ & $2,707^{\mathrm{a}} \pm 0,41$ \\
\hline $\mathrm{FP}\left(\mathrm{mg} \mathrm{dL}^{-1}\right)$ & $153,3^{a} \pm 83,38$ & $150,0^{\mathrm{a}} \pm 51,89$ & $166,7^{\mathrm{a}} \pm 81,65$ & $146,7^{\mathrm{a}} \pm 83,38$ \\
\hline Leucócitos $\left(\times 10^{3} \mathrm{~mm}^{-3}\right)$ & $30,07^{\mathrm{a}} \pm 4,07$ & $35,00^{\mathrm{a}} \pm 4,89$ & $46,47^{b} \pm 11,83$ & $42,00^{\mathrm{b}} \pm 4,03$ \\
\hline Linfócitos $\left(\mathrm{x} 10^{3} / \mathrm{mm}^{-3}\right)$ & $19,50^{\mathrm{a}} \pm 3,90$ & $21,22^{\mathrm{ab}} \pm 4,15$ & $27,39^{\mathrm{c}} \pm 7,18$ & $24,83^{\mathrm{bc}} \pm 6,98$ \\
\hline Heterófilos $\left(\times 10^{3} \mathrm{~mm}^{-3}\right)$ & $7,64^{\mathrm{a}} \pm 2,39$ & $10,24^{\mathrm{b}} \pm 2,18$ & $12,72^{\mathrm{b}} \pm 4,48$ & $11,18^{\mathrm{b}} \pm 2,78$ \\
\hline Monócitos $\left(\mathrm{x} 10^{3} \mathrm{~mm}^{-3}\right)$ & $2,688^{\mathrm{a}} \pm 0,99$ & $3,569^{\mathrm{a}} \pm 1,36$ & $5,497^{b} \pm 2,387$ & $5,740^{\mathrm{b}} \pm 1,98$ \\
\hline Eosinófilos $\left(\mathrm{x} 10^{3} \mathrm{~mm}^{-3}\right)$ & $0,083^{a} \pm 0,18$ & $0,146^{\mathrm{ab}} \pm 0,22$ & $0,406^{b} \pm 0,39$ & $0,204^{\mathrm{ab}} \pm 0,29$ \\
\hline
\end{tabular}

Os dados tabulados estão expressos pela média do grupo \pm desvio padrão (DP) de 15 animais.

a,b,c nas fileiras: médias seguidas por letras diferentes diferem estatisticamente $(\mathrm{P}<0,05)$. PRF (Peso relativo do fígado); VCM (volume celular médio); CHCM (concentração hemoglobina celular média); PPT (proteína plasmática total); FP (fibrinogênio plasmático).

dentro dos índices de normalidade descritos para frangos de corte (JAIN, 1993; NORIEGA, 2000). Todavia, em relação à leucometria global e diferencial, houve aumento significativo no número total e específico dos leucócitos nos grupos inoculados com $2,25 \mathrm{mg} \mathrm{kg}^{-1} \mathrm{e} 4,5 \mathrm{mg} \mathrm{kg}^{-1}$. Com relação ao número de heterófilos, observou-se aumento significativo em todos os grupos testados com piperina quando comparados com o grupo controle. Aparentemente, a administração de piperina por gavagem promoveu estimulação do sistema imunológico das aves, corroborando os resultados obtidos recentemente por SUNILA \& KUTTAN (2004). Esses mesmos pesquisadores demonstraram a capacidade imunomodulatória e antitumoral da piperina em camundongos, observando aumento da celularidade da medula óssea e de células $\alpha$-esterase positivas, aumento do número de leucócitos totais e de células formadoras de placas no baço, promovendo elevação do título de anticorpos circulantes, isto é, da resposta imune humoral.

Em mamíferos, a suplementação oral de piperina promove, dentre outros efeitos, a prevenção de danos celulares, devido ao seu papel anti-oxidativo e anti-apoptótico. A atividade antioxidante direta da piperina contra vários radicais livres foi explorada in vitro por MITTAL \& GUPTA (2000). Recentemente, PATHAK \& KHANDELWAL (2007) demonstraram, além da capacidade anti-oxidativa e anti-apoptótica, que a piperina era capaz de restaurar a capacidade

Tabela 2 - Lesões histopatológicas observadas no fígado de frangos de corte inoculados por via oral com piperina em diferentes concentrações por 14 dias.

\begin{tabular}{lllll}
\hline & & & & \\
& & & \\
Lesões histopatológicas observadas no fígado & 0,00 & 1,12 & 2,25 & 4,50 \\
\hline Degeneração de hepatócitos & - & - & $(+)$ & + \\
Congestão & - & - & $(+)$ & + \\
Infiltrado de células mononucleares & $(+)$ & - & + & + \\
Esteatose & - & - & $(+)$ & + \\
Proliferação dos ductos biliares & - & - & - & + \\
Necrose de ducto biliar & - & & + \\
\hline
\end{tabular}

Legenda: ausência de lesão - ; lesão discreta (+); lesão moderada +; lesão grave ++. 
proliferativa e as alterações fenotípicas (CD4/CD8) de células imunes intoxicadas por cadmium.

A determinação da dose oral segura e a aparente estimulação do sistema imunológico promovido em frangos de corte, aliadas às propriedades biológicas e farmacológicas da piperina já demonstradas em mamíferos, incentivam o estudo dessa amida nesse modelo animal (PIYACHATURAWAT et al, 1983; GUPTA et al., 2000; SUNILA \& KUTTAN, 2004; PATHAK \& KHANDELWA, 2007). No setor avícola, estudos sobre a incorporação de substâncias naturais, como fitoterápicos ou promotores de desempenho alternativos, vêm sendo estimulados, principalmente, estudos com substâncias que aumentem a resistência do organismo animal contra diversas doenças, intoxicações ou adversidades ambientais que comprometem o sistema imunológico das aves.

\section{CONCLUSÕES}

Os resultados obtidos sugerem que a administração oral de $1,12 \mathrm{mg}$ de piperina $\mathrm{kg}^{-1}$ de peso vivo por 14 dias consecutivos não foi tóxica para frangos de corte, promovendo estimulação significativa $(\mathrm{P}<0,05)$ do número de heterófilos. $\mathrm{O}$ aumento da dose oral de piperina em frangos de corte proporcionou alterações histopatológicas dose-dependente nos tecidos analisados e elevação significativa $(\mathrm{P}<0,05)$ do número total e específico de leucócitos.

\section{AGRADECIMENTO}

À Coordenação de Aperfeiçoamento de Pessoal de Nível Superior(CAPES) e ao Conselho Nacional de Desenvolvimento Científico e Tecnológico (CNPq).

\section{COMITÊ DE ÉTICA E BIOSSEGURANÇA}

Este trabalho foi realizado de acordo com as normais éticas, sendo aprovado (processo $n^{\circ}$ 23083.010356/2007-69) pela Comissão de Ética do Instituto de Veterinária/ UFRRJ.

\section{REFERÊNCIAS}

ARAUJO-JUNIOR, J.X. et al. Piperdardina, a piperidine alkaloid from Piper tuberculatum. Phytochemistry, v.44, n.3, p.559561, 1997.

ATAL, C.K. et al. Biochemical basis of enhanced drug bioavailability by piperine: evidence that piperine is a potent inhibitor of drug metabolism. Journal Pharmacology and Experimental Therapeutics, v.232, p.258-262, 1985.

BADMAEV, V. et al. Piperine, an alkaloid derived from black pepper increases serum response of beta-carotene during 14days of oral beta-carotene supplementation. Nutrition Research, v.19, p.381-388, 1999.
BANO, G. et al. Effect of piperine on bioavailability and pharmacokinetics of propanolol and theophylline in healthy volunteers. European Journal of Clinical Pharmacology, v.41 p. 615-617, 1991.

BHAT, B.G.; CHANDRASEKHARA, N. Studies on metabolism of piperine: absorption, tissue distribution and excretion of urinary conjugates in rats. Toxicology, v.40, p.83-92, 1986.

CARDOSO, V.S. Efeitos da piperina em frangos de corte (Gallus gallus) com intoxicação experimental por aflatoxinas. 2007. 47f. Dissertação (Microbiologia Veterinária) - Curso de Pós-graduação em Microbiologia Veterinária, Universidade Federal Rural do Rio de Janeiro.

CAMPBELL, T.W.; DEIN, F.J. Avian hematology. The basics. Veterinary Clinics of North America Small Animal Practice, v.14, n.2, p.223-248, 1984.

COLES, E.H. Patologia clínica veterinária. 3.ed. São Paulo: Manole, 1984. 566p.

DOGRA, R.K.S. et al. Immunotoxicological effects of piperine in mice. Toxicology, v.196, n.3, p.229-36, 2004. Disponível em: <http:/ /www.sciencedirect.com/science?_ob=ArticleURL\&_udi=B6TCN4BRJ5Y9-7\& user $=687350 \&$ coverDate $=03 \% 2 \mathrm{~F} 15 \% 2 \mathrm{~F}$ $2004 \&$ \& 1 i d = 831554077 \&_rd o c $=2$ \&_ $\mathrm{fmt}=$ high $\&$ \&_orig $=$ search $\&$ _cdi $=5175 \&$ \&_sort $=$ d \&_doc anchor $=\&$ view $=$ c\&_ct $=3 \&$ \&acct $=$ C000037881\&_version $=1 \&$ \&urlVe rsion=0\&_userid=687350\&md5=2701231a70e77641f03f6f197bf7b993>. Doi:10.1016/j.tox.2003.10.006.

ESTRELA, J.L.V. et al. Toxicidade de amidas análogas à piperina a larvas de Ascia monuste orseis Godart (Lepdopitera: Pieridae) e Spodoptera frugiperda (J. E. Smith) (Lepidóptera: Noctuidae). Neotropical Entomology, v.32, n.2, p.343-346, 2003. Disponível em: <http://www.scielo.br/scielo.php?script=sci_arttext\&pid=S1519566X2003000200022>. Doi: 10.1590/S1519-566X2003000200022.

GHOSHAL, S. et al. Antiamoebic activity of Piper longum fruits against entamoeba histolytica in vitro and in vivo. Journal of Ethnopharmacology, v.50, p.167-170, 1996. Disponível em: < h t t p : / / w w w . s c i e n c e d i r e c t . c o m/ science?_ob=ArticleURL\&_udi=B6T8D-45W3WMV$6 \&$ _user $=687350 \&$ _coverDate $=03 \% 2 F 31 \% 2 F 1996 \&$ alid $=83155$ $4343 \&$ \&doc $=1 \&$ \&mt $=$ high \&_orig =search\&_cdi $=5084 \&$ sort $=$ d\&_docanchor $=\&$ view $=c \&$ ct $=1 \&$ \& acct $=$ C 0 $0000378881 \&$ \&_vers i o n $=1$ \&_u r l Vers i on $=0 \&$ _userid $=687350 \& \mathrm{md} 5=0 \mathrm{~b} 303 \mathrm{dd} 264 \mathrm{c} 3320 \mathrm{~d} 593 \mathrm{c} 732 \mathrm{ed} 13 \mathrm{a} 00 \mathrm{cb}>$. Doi: 10.1016/0378-8741(96)01382-7.

GUPTA, S.K. et al. Comparative anti-nociceptive, antiinflammatory and toxicity profile of nimesulide vs nimesulide and piperine combination. Pharmacological Research, v.41, n.6, p.657-662, 2000.

IKAN, R. In natural products. A laboratory guide. 2.ed. New York: Academic, 1991. p.233-238.

JAIN, N.C. Comparative hematology features of some avian and mammalian species. In: __ Essential of veterinary hematology. Philadelphia: Lea \& Febiger, 1993. Cap.3, p. 54-71

LUNA, L.G. Manual of histologic staining methods of the Armed Forces Institute of Pathology. 13.ed. New York: McGraw-hill, 1968. 258p. 
MALINI, T. et al. Effects of piperine on testis of albino rats. Journal Ethnopharmacology, v.64, p.219-225, 1999.

MITTAL, R.; GUPTA, R.L. In vitro antioxidant activity of piperine. Methods and Findings in Experimental and Clinical Pharmacology, v.22, p.271-274, 2000.

NATT, M.P.; HERRICK, C.A. A new blood diluent for counting the erythrocytes and leucocytes of the chicken. Poultry Science, v.31, p.735-738, 1951.

NORIEGA, M.L.V.C. Apuntes de hematología aviar: material didático para curso de hematologia aviária. México: Universidad Nacional Autónoma de México. Departamento de produccíon animal: aves, 2000. 70p.

PATHAK, N.; KHANDELWA, S. Cytoprotective and immunomodulating properties of piperine on murine splenocytes: an in vitro study. European Journal of Pharmacology, v.576, p.160-170, 2007. Disponível em: <http:// www.sciencedirect.com/ science?_ob=ArticleListURL\&_method=list\&_ArticleListID=83 $1545755 \&$ \&_s ort $=$ d \& vi e w $=$ c \&_a c c t $=$ C 00003 $7881 \&$ \&_version $=1 \&$ _urlversion $=0 \&$ \& useri $\mathrm{d}=687350 \&$ md5=784a53f9d5bcee657006b9c0950c436d $>$. Doi: 10.1016/j.ejphar.2007.07.033.

PARMAR, V.S. et al. Phytochemistry of the genus piper. Phytochemistry, v.46, p.597-673, 1997. Disponível em: <http:// www.sciencedirect.com/science?_ob=ArticleListURL\&_method=list \&_ArticleListID $=831549202 \& \_$sort $=$d \&view $=$c\&_acct $=$C0000 37881\&_version=1\&_urlVersion=0\&_userid $=687350 \&$ md5 $=32006$ 767c68838985bbf9b233619db91>.Doi:10.1016/S00319422(97)00328-2.

PIYACHATURAWAT, R. et al. Acute and subacute toxicity of piperine in mice, rats and hamsters. Toxicology Letter, v.16, p.351-359, 1983.

PRADEEP, C.R.; KUTTAN, G. Effect of piperine on the inhibition of lung metastasis induced by B16F-10 melanoma cells in mice. Clinical and Experimental Metastasis, v.19, p.703-708, 2002.

REEN, K.R. et al. Piperine inhibits aflatoxin B1-induced cytotoxicity and and genotoxicity in V79 Chinese hamster cells genetically engineered to express rat cytochrome P4502B1. Journal of Ethnopharmacology, v.58, p.165173, 1997. Disponível em: <http://www.sciencedirect.com/
science?_ob=ArticleURL\&_udi=B6T8D-3RV5TV0 $4 \&$ user $=687350 \&$ _coverDate $=11 \% 2 \mathrm{~F} 30 \% 2 \mathrm{~F} 1997 \&$ \&alid $=8315$ 49640\&_rdoc $=18$ \&_fmt $=$ high\&_orig $=$ search\&_cdi $=5084 \&$ _ s o r t $=$ d \&_d o c a n c h or $=\&$ vi e w $=$ c \& $\&$ c t $=2 \&$ _acct $=$ C000037881\&_version $=1 \&$ \&urlVersion $=0$ \&_userid $=687350$ \&md5=b8d6d6bffd7a6a08f0bd23add880916f>. Doi: 10.1016/ S0378-8741(97)00104-9.

ROSTAGNO H.S. Tabelas brasileiras para aves e suínos. Composição de alimentos e exigências nutricionais. 2.ed. Viçosa: UFV, 2005. 186p.

SELVENDIRAN, K. et al. Protective effect of piperine on benzo(a)pyrene-induced lung carcinogenesis in Swiss albino mice. Clinic Chimica Acta, v.50, p.3-78, 2004.

SHOBA, G. et al. Influence of piperine on the pharmacokinetics of curcumin in animals and human volunteers. Planta Medica, v.64, p.353-356, 1998.

SIDDIQUI, B.S. et al. An amide from fruits of Piper nigrum. Phytochemistry, v.45 n.8, p.1617-1619, 1997. Disponível em: <http://www.sciencedirect.com/ science?_ob=ArticleURL\&_udi=B6TH7-3SB1JWN$27 \&$ \&user $=687350 \&$ \&coverDate $=08 \% 2$ F31\%2F $1997 \&$ _alid $=831551082 \&$ \& rdoc $=4 \&$ \& $\mathrm{mt}=\mathrm{high} \&$ \&orig $=$ search \& _c di $=5275 \&$ \&_sort $=$ d \&_d o c a n c hor $=\&$ vi e w $=$ c\&_ct $=9 \&$ \&_acct $=\mathrm{C} 000037881 \&$ _version $=1 \&$ \&_urlVersion $=$ 0\&_userid=687350\&md5=dbf0bf1349c2e8467a8a894c05223801 >. Doi: 10.1016/S0031-9422(97)00202-1.

SUNILA, E.S.; KUTTAN, GJ. Immunomodulatory and antitumor activity of Piper longum Linn. and piperine. Journal Ethnopharmacology, v.90, n.2-3, p.339-46, 2004. Disponível em: <http:// www.sciencedirect.com/science?_ob=ArticleURL\&_udi=B6T8D4 B66CD $1-1 \&$ \&_user $=687350 \&$ \&_coverDate $=02 \% 2$ F $29 \% 2 \mathrm{~F}$ $2004 \&$ \&alid $=831553307 \&$ \&doc $=18$ \& fmt $=$ high\&_orig $=$ searc h \&_ c d i $=5084 \&$ _ s ort $=$ d \&_d o c a n c h or $=\&$ v

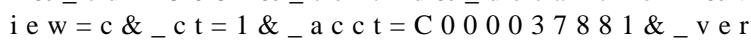
s i o $n=1 \&$ \& r l V e r s i o n $=0$ \& _ u s e r i $\mathrm{d}=687350 \& \mathrm{md} 5=843 \mathrm{e} 9 \mathrm{cdd} 39 \mathrm{ac} 71 \mathrm{a} 8568 \mathrm{ae} 8 \mathrm{ea} 230 \mathrm{~b} 4 \mathrm{ff} 3>$. Doi:10.1016/j.jep.2003.10.016.

WINTROBE, M.M. Variations in the size and hemoglobin content of eritrocytes in the blood of various vertebrates. Folia Haematologica. Internationales Magazin fur Slutforschung, Leipzig, v.51, p.31, 1933. 